\title{
U so de factor VII en el control de sangrados perioperatorios: Evidencias actuales
}

\author{
Rodrigo López B, N icolás Aeschlimann D, Claudia Carvajal F, \\ G uillermo Lema $F$.
}

\section{Recombinant activated factor VII for perioperative bleeding}

Recombinant activated factor VII (rFVIIa) is a new hemostatic drug, originally used for the treatment of patients with hemophilia A and B. At the present time it is used for other bleeding conditions such as the perioperative period. When used prophylactically there is a reduction in the number of bleeding episodes but no changes in the need for blood transfusion or other blood products. The adverse effects are arterial or venous thromboembolic events that are mostly related to the severity of the underlying disease of the patient and the concurrent administration of other hemostatic agents, rather than the use of rFVIIa. Its use is recommended when there is a persistent bleeding after the reposition of blood products and when surgical causes of bleeding have been discarded. The cost of the medication should also be considered before its use (Rev Méd Chile 2009; 137: 837-43).

(Key words: Blood component transfusion; Factor VII a; Postoperative hemorrhage)

Recibido el 6 de marzo, 2008. Aceptado el 23 de septiembre, 2008.

Departamento de Anestesiología, Hospital Clínico Pontificia Universidad Católica de Chile. Santiago de Chile

$\mathrm{E}$ Factor VII (FVII) es un potente agente prohemostático. Su uso clínico se ha estudiado desde comienzos de la década $1980-89^{1}$, pero esos estudios se vieron limitados por la difícil obtención del factor aislado. Fue el lanzamiento de la forma recombinante (rFVII) en 1988 el que facilitó su uso clínico, que fue aprobado por la Food and Drugs Administration (FDA) de los Estados Unidos de Norteamérica (EE.UU.) para el tratamiento de episodios de sangrado en pacien-

$\overline{\text { Correspondencia a: Dr. Rodrigo López B. Departamento de }}$ Anestesiología, Pontificia Universidad Católica de Chile. Marcoleta 367. Fono: 3543415. E mail: ralopez@puc.cl tes con hemofilia A y B con inhibidores del factor VIII o IX, respectivamente. En 2005 se amplió la autorización al incluir cirugías de los pacientes antes mencionados y episodios de sangrado en pacientes con déficit de factor VII. En Europa su uso está aprobado, además, para pacientes con trombastenia de Glanzmann refractarios a transfusiones de plaquetas ${ }^{2}$.

Para entender la manera en que este factor actúa, es necesario hacer referencia a algún modelo de hemostasia. El paradigma clásico o "cascada" de la coagulación se ve cuestionado por la separación entre la vía extrínseca y la vía intrínseca, así como por la falta de participación de plaquetas y otros elementos celulares. Así, se ha postulado el "modelo celular" de la coagula- 
ción, que constaría de 3 fases: Iniciación, en que el FT se une a FVIIa con la producción de pequeñas cantidades de trombina determinando activación plaquetaria; Amplificación, formándose complejos $\mathrm{FVa} / \mathrm{FXa}$ sobre la superficie de las plaquetas activadas; y Propagación ${ }^{4}$, en que los complejos $\mathrm{FVa} / \mathrm{FXa}$ producen una explosión de trombina.

A partir de este nuevo paradigma se entienden algunos fenómenos de la hemostasia como el efecto del factor VII recombinante activado (rFVIIa). Las dosis utilizadas en clínica elevan las concentraciones plasmáticas de FVIIa por sobre 500 veces las fisiológicas, con lo que no sería necesaria la presencia de FT para catalizar la activación del FX, sino que lo hace unido a factores presentes en la superficie de las plaquetas $\operatorname{activadas}^{3,4}$

Es posible, entonces concluir que:

a. Para que el rFVIIa sea efectivo en la hemostasia es necesario que existan concentraciones significativas de plaquetas y de factores de coagulación como el FX, el FV, protrombina y fibrinógeno

b. No sólo se necesitan concentraciones apropiadas, sino que además deben existir las condiciones del medio apropiadas (temperatura y $\mathrm{pH}$, entre otras.)

Desde su aprobación por la FDA en 1999, ha aumentado de manera considerable el uso de rFVIIa. En EE.UU, en el año 2000 se utilizó en menos de 500 pacientes, en 2004 más de 4.000 pacientes. Este mayor uso se relaciona probablemente con un menor costo y mayor disponibilidad, pero también se debe a una mayor cantidad de indicaciones, que incluyen usos no autorizados. Se estima que en 2004 se utilizó rFVIIa en más de 80 patologías diferentes, estando aprobado su uso en ese momento sólo para pacientes con hemofilia A y B con los inhibidores respecti$\operatorname{vos}^{2}$. Entre estos diagnósticos hay que mencionar: otros trastornos de la coagulación, sangrado en pacientes con alteraciones de la función hepática, reversión de anticoagulación (por antagonistas de vitamina $\mathrm{K}$, heparina de bajo peso molecular, pentasacáridos, etc.) o episodios hemorrágicos importantes, sean traumáticos o postoperatorios ${ }^{5}$.

Debido a la relevancia de los sangrados perioperatorios y a la gran heterogeneidad de los reportes de rFVIIa en este contexto, el presente trabajo tiene como objetivo revisar la información sobre este uso particular.

\section{USOS: SANGRADO PERIOPERATORIO}

Existen numerosos reportes de su utilización, con muy pocos trabajos prospectivos que avalen su uso. El ensayo clínico aleatorio más citado en la literatura se realizó en pacientes sometidos a prostatectomía radical y demostró una reducción del sangrado de 50\% al utilizar dosis de $40 \mu \mathrm{g} / \mathrm{Kg}$ comparados con el grupo placebo; ninguno de estos pacientes requirió transfusiones de derivados sanguíneos. Al utilizar dosis de $20 \mu \mathrm{g} / \mathrm{Kg}$ se disminuyó el sangrado en 35\% comparado con el grupo placebo, y sólo 38\% de los pacientes necesitó de transfusiones de glóbulos rojos, comparado con 60\% en el grupo placebo ${ }^{6}$.

Uno de los grupos de pacientes en el que resulta particularmente atractivo el potencial uso de rFVIIa son los sometidos a cirugía de escoliosis, ya que éstos frecuentemente presentan sangrado importante. Pese al uso de numerosas técnicas de ahorro transfusional actualmente utilizadas, se estima que durante la cirugía se podría perder $20 \%$ de la volemia; en los pacientes que padecen enfermedades neuromusculares esta pérdida varía entre $25 \%$ y $127 \%$ de la volemia. El sangrado postoperatorio también debe ser considerado, y se estima que en 20\% de los pacientes éste excede a las pérdidas del intraoperatorio. En este grupo de pacientes se evaluó la utilidad de la administración profiláctica de $23 \mu \mathrm{g} / \mathrm{Kg}$ de rFVIIa en pacientes sin alteraciones de la coagulación previa. Si bien disminuye de manera significativa el sangrado intraoperatorio, no disminuyó el sangrado postoperatorio, ni la necesidad de transfusión de glóbulos rojos o plasma fresco congela$\mathrm{do}^{7}$.

Los pacientes sometidos a cirugía hepática también son un grupo potencialmente beneficiado con el uso de rFVIIa, ya que se estima que hasta $40 \%$ de estos pacientes requieren reposición con hemoderivados. En un estudio que incluyó a 185 pacientes sometidos a resección de al menos 3 segmentos hepáticos, sin daño hepático previo, se constató una tendencia a menor sangrado con el uso profiláctico de rFVIIa, con una reducción en 
la caída del hematocrito durante la cirugía, pero sin diferencias significativas en el sangrado postoperatorio ni en la cantidad de glóbulos rojos transfundidos 8 .

Otro tipo de cirugía que expone a los pacientes a un riesgo de sangrado considerable es la cirugía cardiaca. Se estima que hasta $15 \%$ de la sangre donada a bancos de sangre es utilizada en este grupo de pacientes, y hasta $80 \%$ de los pacientes necesitarán transfusión de hemoderivados 9 . En un estudio en que se evaluó el uso profiláctico de rFVIIa en pacientes sometidos a cirugía cardiaca, se encontró una disminución en la cantidad de glóbulos rojos transfundidos y en la cantidad de plasma fresco congelado administrado, lo que se tradujo en una disminución del total de unidades de hemoderivados transfundidas de 105 en el grupo control a 13 en el grupo rFVIIa. Sin embargo, en este análisis fue excluido un paciente en el grupo rFVIIa que presentó un sangrado quirúrgico, causa frecuente en este tipo de cirugías. Al analizar los resultados según "intención de tratar", las diferencias encontradas pierden magnitud: en el caso de los glóbulos rojos se alcanza un valor $\mathrm{p}$ de 0,11 , para el plasma fresco congelado un valor $\mathrm{p}$ de 0,18 , y el total de unidades de hemoderivados utilizados fue de 105 en el grupo control v/s 74 en el grupo rFVIIa con una significación estadística de $0,052^{9}$.

Existen numerosos reportes de uso de rFVIIa en pacientes testigos de Jehová sometidos a cirugía cardiaca en que se no se desea administrar hemoderivados. Si bien estos reportes son exitosos, reflejan un uso muy particular en casos excepcionales.

En el ámbito de neurocirugía es donde se ha intentado con mayor fuerza encontrar un rol al rFVIIa debido a las consecuencias deletéreas que los sangrados tienen en este grupo de pacientes. Se suele argumentar que posee un inicio de acción más rápido, sin la necesidad de someter al paciente a grandes sobrecargas de volumen y que es efectivo incluso cuando otras medidas han fallado; sin embargo, pese a sus potenciales beneficios, hasta el momento para patologías como el trauma encefálico o el sangrado perioperatorio sólo existen reportes de casos en los que disminuiría el sangrado hasta en 59\%. En la hemorragia subaracnoídea, en donde otros agentes prohemostáticos disminuirían el riesgo de sangrado a costa de un mayor riesgo de hidrocefalia, vasoespasmo e infarto, se diseñó un estudio usando rFVIIA, que fue suspendido antes de terminar porque un paciente presentó un accidente vascular oclusivo de la arteria cerebral media contralateral al aneurisma ${ }^{10}$. En donde sí se ha podido encontrar una utilidad demostrada al rFVIIa es en las hemorragias intracerebrales. En un estudio se compararon las imágenes de la tomografía axial computarizada (TAC) al ingreso y a las $24 \mathrm{~h}$ de administrada la dosis de rFVIIa. En los pacientes del grupo control el hematoma aumentó de tamaño en 26\%; en los pacientes que recibieron este agente prohemostático, los aumentos de tamaño fueron considerablemente menores, según la dosis administrada (20 $\mu \mathrm{g} / \mathrm{Kg}$ : 18\%; $80 \mu \mathrm{g} / \mathrm{Kg}: 11 \%)$, sin embargo, esto no se reflejó en un efecto significativo sobre las secuelas posteriores ${ }^{11}$

Resulta atractiva la utilización de rFVIIa en las pacientes obstétricas, ya que se registran sangrados masivos con riesgo vital hasta en 1 de 1.000 partos. Se estima que pese a las múltiples estrategias de tratamiento, cada año mueren 125.000 mujeres por esta causa. Debido a la particular situación clínica en que se ha utilizado el rFVIIa, no es posible realizar estudios prospectivos, y las series retrospectivas que han sido publicadas tienen un bajo número de casos. Sin embargo, en prácticamente todos los casos de sangrado por coagulopatía, su uso ha sido exitoso, siempre y cuando exista un correcto diagnóstico etiológico. En una de la series más grandes (12 pacientes) los casos en que el sangrado persistió pese al uso de rFVIIa, respondieron a la embolización de arterias uterinas, por lo que se plantea que correspondían a sangrados quirúrgicos ${ }^{12}$.

\section{MEDICIÓN DEL EFECTO}

Muchos de los trabajos diseñados para evaluar la efectividad clínica del uso de rFVIIa usan pruebas de laboratorio para medir el efecto. El INR sufre modificaciones significativas ${ }^{13}$, las que comienzan a los 15 min luego de administrada la droga y perduran más allá de su vida media ${ }^{7}$. Este comportamiento ha generado la costumbre de utilizar el TP/INR como un indicador de la actividad del rFVIIa; sin embargo, este test frecuentemente 
sobreestima el efecto, por lo que no debería utilizarse con este fin ${ }^{14}$. Ninguna de las pruebas de laboratorio utilizadas en clínica resultan útil para monitorizar la activad del rFVIIa, sino que se debe realizar una apreciación clínica del sangrado antes y después de administrada la droga en conjunto con los requerimientos de hemoderivados.

\section{Efectos ADVERSOS}

Los efectos adversos "leves", con incidencia menor de 1:1.000, incluyen fiebre, dolor en sitio de inyección, cefalea, hipertensión, hipotensión, náuseas, vómitos, edema y eritema cutáneo.

Los efectos adversos "serios", con una incidencia menor de 1:10.000, se relacionan teóricamente con la presencia de factor tisular expuesto anormalmente en el endotelio enfermo (placas de ateroma) con el consecuente riesgo de trombosis arterial o venosa, como con la presencia anormal de factor tisular disuelto en el plasma (sepsis, retiro de circulación extracorpórea) con el consecuente riesgo de coagulación intravascular diseminada.

Algunos de los trabajos diseñados para evaluar la efectividad clínica del rFVIIa evalúan también la presencia de complicaciones. Así, en cirugía hepática no se encontró ninguna diferencia en la ocurrencia de complicaciones tromboembólicas ni en las complicaciones hemorrágicas ${ }^{8}$.

En cirugía cardiaca, un estudio retrospectivo evaluó las complicaciones trombóticas relacionadas con el uso precoz de rFVIIa (antes de 8 unidades de glóbulos rojos transfundidas), con el uso tardío (después de 8 unidades transfundidas) del mismo y con un grupo control. Si bien en el análisis preliminar pareciera que el uso de rFVIIa aumenta el riesgo de complicaciones trombóticas (riesgo combinado: 23,8\% grupo control v/s 30\% grupo uso precoz v/s 60,4\% grupo uso tardío, p $=<0,0001$ ), cuando se realiza un ajuste por variables en que se anula el efecto de factores como la edad, peso, sexo, enfermedad cerebrovascular previa, salida difícil de circulación extracorpórea (CEC) y duración de CEC en el aumento de riesgo de morbilidad, se observa que del uso de rFVIIa no se derivan riesgos aumentados de complicaciones trombóticas en comparación con los pacientes en que no se utilizó (OR 1,04 [0,6-1,8]). En los pacientes en que se utilizó el rFVIIa de manera precoz, se observó una disminución del riesgo de complicaciones en comparación con los pacientes en que se administró de manera tardía (OR 0,41 $[0,18-0,92])$. Se cree que las complicaciones trombóticas están más relacionadas con factores propios del paciente que con el uso de rFVIIa, sin embargo, en caso que se decida utilizar esta droga, es mejor hacerlo de modo precoz ${ }^{15}$.

Las complicaciones trombóticas ocurren en territorio arterial $(54,1 \%)$ y venoso $(40,4 \%)$. Entre los reportes de trombosis arteriales se incluyen accidentes vasculares encefálicos no hemorrágicos, síndromes coronarios agudos y de otras arterias (femoral, hepática, pulmonar, renal, esplénica e ilíaca); las complicaciones venosas incluyen trombosis venosa profunda (extremidades, yugular, mesentéricas, portal, renal y retinal), así como tromboembolismo pulmonar. Otros sitios en que se han reportado trombosis incluyen oxigenadores de circulación extracorpórea (CEC), circuitos de diálisis y tubos endotraqueales ${ }^{3}$.

\section{RECOMENDACIONES}

Existen guías prácticas y recomendaciones en torno a la utilización de rFVIIa en usos no aprobados por la FDA. Estas recomendaciones se basan principalmente en reportes de casos, con un posible sesgo de selección y una la alta heterogeneidad de los trabajos publicados. De este modo, se considera que se basan en evidencia clase $\mathrm{E}$.

Las guías norteamericanas ${ }^{17}$ se orientan principalmente a la utilidad del rFVIIa según el tipo de cirugía, estableciendo que en determinados casos la medida sería "apropiada", considerando estas sugerencias no como una indicación mandatoria sino como un caso en que los potenciales beneficios superarían los efectos adversos, y resultan en consecuencia, aceptables (Tabla 1).

Las recomendaciones europeas ${ }^{14}$ establecen un algoritmo de trabajo (Figura 1) que se basa en 6 principios:

1. Se debe intentar el control del sangrado de manera convencional.

2. El uso de derivados sanguíneos no debe ser reemplazado por rFVIIa. 
Tabla 1. Recomendaciones para el uso no autorizado de rFVII ${ }^{13}$

\section{Terapia de rescate para pacientes quirúrgicos}

Cirugía cardiaca sin reposición adecuada

Cirugía cardiaca luego de reposición adecuada

Cirugía de aorta torácica sin reposición adecuada

Cirugía de aorta torácica luego de reposición adecuada

Cirugía de aorta abdominal sin reposición adecuada

Cirugía de aorta abdominal luego de reposición adecuada

Cirugía hepática sin reposición adecuada

Cirugía hepática luego de reposición adecuada

Cirugía de columna sin reposición adecuada

Cirugía de columna luego de reposición adecuada

Cirugía de reemplazo articular sin reposición adecuada

Cirugía de reemplazo articular luego de reposición adecuada

Hemorragia post parto sin reposición adecuada

Hemorragia post parto luego de reposición adecuada
Inapropiada

Apropiada

Inapropiada

Apropiada

Inapropiada

Incierto

Incierto

Apropiada

Inapropiada

Apropiada

Inapropiada

Inapropiada

Inapropiada

Apropiada

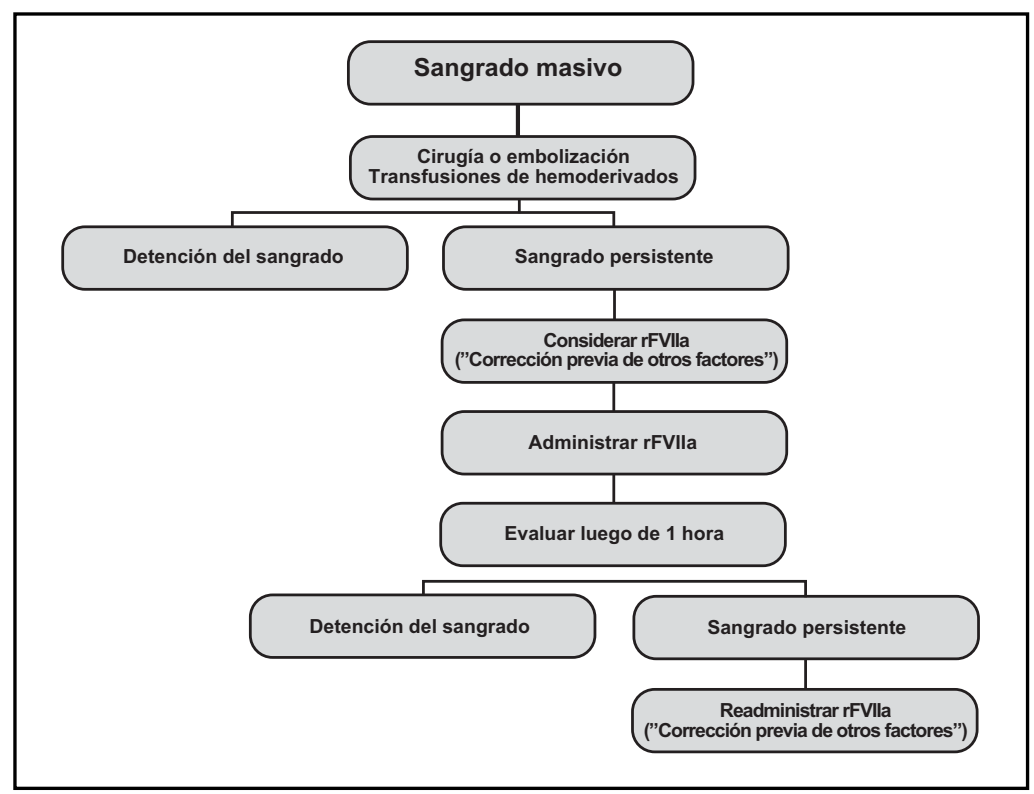

Figura 1. Algoritmo de trabajo de las recomendaciones europeas para el uso de rFVIIa ${ }^{14}$.

"Corrección previa de otros factores" se entiende por: hematocrito $\geq 24 \%$, fibrinógeno 50-100 mg/dL, plaquetas $\geq 50000$ y $\mathrm{pH}>7,2$.

3. Se debe corregir otros factores que afectan la coagulación (hipotermia, acidosis, anemia, calcemia).

4. Luego, se puede considerar el uso de rFVIIa.
5. Se debe contar con el consentimiento del paciente o algún representante debido a que se trata de un uso no autorizado.

6. No se debe utilizar rFVIIa de manera profiláctica. 
Las guías de uso de rFVIIa israelíes ${ }^{18}$ provienen de un contexto diferente, y aunque están originadas en relación a la experiencia militar, incluyen algunas consideraciones sobre pacientes quirúrgicos. Una vez superada la fase inicial de reanimación, consideran el uso de rFVIIa en "cualquier paciente 'salvable' que sufre de un sangrado masivo e incontrolable, que no responda a las medidas quirúrgicas y de reposición apropiadas", definiendo sangrado masivo como 100\% de la volemia en $24 \mathrm{~h}, 50 \%$ de la volemia en $3 \mathrm{~h}$, un sangrado mayor a $150 \mathrm{ml} / \mathrm{min}$ o un sangrado mayor a $1,5 \mathrm{ml} / \mathrm{Kg} / \mathrm{min}$ por más de $20 \mathrm{~min}$. Entienden por medidas quirúrgicas apropiadas todas las medidas disponibles y aceptables en la situación determinada, y por medidas de reposición apropiadas la administración de plasma fresco congelado (10-15 ml/Kg), crioprecipitados (1-2 U cada $10 \mathrm{Kg}$ ) o plaquetas (1-2 U cada $10 \mathrm{Kg}$ ), buscando concentraciones de fibrinógeno mayores a $50 \mathrm{mg} / \mathrm{dL}$ y de plaquetas mayores a $50.000 / \mathrm{ml}$, en condiciones de normalidad acido-base $(\mathrm{pH}>7,2)$ y normotermia. De acuerdo con estas guías, estaría contraindicado de manera absoluta en caso de paciente no 'salvable'; la historia de trombosis arterial o venosa en un lapso menor a 6 meses sería una contraindicación relativa. Entre las consideraciones quirúrgicas se menciona el retiro del "paking" previo a la administración de rFVIIa y la consideración de una angiografía o una revisión en "segundo tiempo", con el fin de descartar una causa quirúrgica que explique el sangrado.

Las políticas de uso de rFVIIa canadienses ${ }^{19}$ proponen lineamientos similares en cuanto a la administración de esta droga una vez realizadas las medidas de reposición adecuadas, con exámenes de laboratorio en los rangos antes descritos y asegurándose de utilizar dosis suficientes (20-50 $\mu \mathrm{g} / \mathrm{Kg}$ ); sugieren la implementación de un sistema de registro que permita la notificación de eventos adversos.

\section{DATOS PRÁCticos}

La dosis a administrar en casos de pacientes quirúrgicos aún es materia de debate. Si bien se han obtenido buenos resultados con cantidades tan bajas como 5 $\mu \mathrm{g} / \mathrm{Kg}$, otros protocolos consideran la administración de hasta $300 \mu \mathrm{g} / \mathrm{Kg}$. Las recomendaciones sugieren utilizar dosis similares a las utilizadas en pacientes con hemofilia, 90 a $120 \mu \mathrm{g} / \mathrm{Kg}^{14,17,18}$. El efecto clínico ocurre a los 10-20 min, y en casos de persistencia del sangrado se podría repetir la misma dosis. Es necesario recordar que la vida media de este fármaco es breve $(2 \mathrm{~h})$, por lo que eventualmente sería necesario repetir la administración de rFVIIa. Está contraindicado en pacientes con reacciones conocidas de hipersensibilidad a proteínas de murinas o bovinas, debido a que podrían estar presentes rastros de proteínas de estos animales producto del proceso de amplificación y purificación. Se desconocen efectos en el embarazo y lactancia, por lo que debe primar el juicio clínico en relación al riesgo/beneficio.

Uno de los elementos a tener en consideración al momento de decidir la utilización de rFVIIa es el costo, y si bien su uso permite un ahorro en hemoderivados, éste sería de menor cuantía?. Es posible que la administración de este medicamento en dosis única sea recomendable desde el punto de vista costo-efectividad en pacientes que presentan un sangrado postrauma ${ }^{20}$; en el contexto quirúrgico se estima que esta condición se alcanza sólo si permite un ahorro estimado de 50 unidades de glóbulos rojos (o su equivalente en otros hemoderivados), de 2 días de UCI o un procedimiento de embolización de urgencia ${ }^{12}$.

\section{Conclusiones}

El rFVIIa es un potente agente prohemostático. No se ha podido demostrar su utilidad al administrarlo de manera profiláctica, incluso en cirugías en que el sangrado sigue siendo un problema, como son la cirugía de resección hepática, la artrodesis de columna y la cirugía cardiaca con circulación extracorpórea. Sin embargo, constituye una droga útil cuando se trata de un sangrado no quirúrgico que no ha respondido a las medidas de reposición apropiadas. Debido a la falta de estudios adecuados, es difícil determinar la real incidencia de complicaciones derivadas del uso de rFVIIa; el riesgo teórico de trombosis arterial y venosa que tendrían estos pacientes, estaría relacionado con las patologías del paciente y con el uso de otros agentes prohemostáticos. El costo de este fármaco es un factor a considerar, por lo que su uso debiera analizarse caso a caso, o bien, regularse por un protocolo de manejo de sangrado perioperatorio. 


\section{REFERENCIAS}

1. Hedner U, Kisiel W. Use of factor Vila in the treatment of two hemophilia A patients with hightiter inhibitors. J Clin Invest 1983; 71: 1836-41.

2. O'Connell KA, Wood JJ, Wise RP, Lozier JN, Braun MM. Thromboembolic Adverse Events After Use of Recombinant Human Coagulation Factor VIIa. JAMA 2006; 295: 293-8.

3. Lisman T, De Groot PG. Mechanism of action of recombinant factor VIIa. Journal of Thromb Haemost 2003; 1: 1138-9.

4. Becker M. Cell-Based Models of Coagulation: A Paradigm in Evolution. Journal of Thrombosis and Thrombolysis 2005; 20: 65-8.

5. Levi M, Peters M, Büller HR. Efficacy and safety of rFVIIa for the treatment of severe bleeding: A systematic review. Crit Care Med 2005; 33: 883-90.

6. Friederich PW, Henny CP, Messelink EJ, Geerdink MG, Keller T, Kurth KH ET al. Effect of rFVIIa on perioperative blood loss in patients undergoing retropubic prostatectomy. Lancet 2003; 361: 201-5.

7. Kolban M, Balachwska-Kosciolek I, Chmielnicki M. Recombinant coagulation FVIIa-a novel haemostatic agent in scoliosis surgery? Eur Spine J 2006; 15: 944-52.

8. Lodge JP, Jonas S, Oussoultzoglou E, Malagó M, Jayr C, Cherqui D et al. rFVIIa in Major Liver Resection. Anesthesiology 2005; 102: 269-75.

9. Diprose P, Herbertson MJ, O'Shaughnessy D, Gill RS. rFVIIa after $\mathrm{CPB}$ reduce allogenic transfusion in complex non-coronary cardiac surgery: randomized double-blind placebo-controlled pilot study. $\mathrm{Br} \mathrm{J}$ Anaesth 2005; 95: 596-602.

10. Hawryluk G, Cusimano MD. The role of rFVIIa in neurosurgery: hope or hype? J Neurosurg 2006; 105 : 859-68.
11. Mayer S, Brun N, Begtrup K, Broderik J, Davis S, Diringer $\mathrm{M}$ et al. Efficacy and Safety of rFVIIa for Acute Intracerebral Hemorrhage. N Engl J Med 2008; 358: 2127-37.

12. Karalapillai D, Popham P. rFVIIa in massive postpartum haemorrhage. Int J Obstet Anesth 2007; 16: 29-34.

13. Bishop C, Renwick We, Hogan C, Haeusler M, TucKField A, Tatoulis J. rFVIIa: Treating Postoperative Hemorrhage in Cardiac Surgery. Ann Thorac Surg 2006; 81: 875-9.

14. Vincent JL, Rossaint R, Riou B, Ozier Y, Zideman D, SPAHN DR. Recommendations on the use of rFVIIa as an adjunctive treatment for massive bleeding -a European perspective. Critical Care 2006; 10: R120.

15. Karkouti K, Yau TM, Riazi S, Dattilo KM, Wasowicz M, Meineri M et AL. Determinants of complications with rFVIIa for refractory blood loss in cardiac surgery. Can J Anesth 2006; 53: 802-9.

16. Warren O, Mandal K, Hadjianastassiou V, Knowlton L, PANESAR S, John K ET AL. rFVIIa in Cardiac Surgery: A Systematic Review. Ann Thorac Surg 2007; 83: 707-14.

17. Shander A, Goodnough lT, Ratko T, Matuszewsik K, Cohn S, Diringer M et al. Consensus Recommendations for the Off-Label Use of rFVIIa Therapy. Pharmacy \&Therapeutics 2005; 30: 641-58.

18. Martinowitz U, Michaelson M. Guidelines for the use of rFVIIa in uncontrolled bleeding: a report by the Israeli Multidisciplinary rFVIIa Task Force. J Thromb Haemost 2005; 3: 640-8.

19. Moltzan CJ, Anderson A, Callum J, Fremes S, Hume $\mathrm{H}$, Mazer $\mathrm{D}$ et al. The evidence for the use of rFVIIa in massive bleeding: development of a transfusion policy framework. Transf Med 2008; 18: 112-20.

20. Morris S, Ridley S, Munro V, Christensen MC et al. Cost effectivness of rFVIIa for the control of bleeding in patients with severe blunt trauma injuries in the United Kingdom. Anaesth 2007; 62: 43-52. 